\title{
Retraction
}

\section{Retracted: Inflammatory Microenvironment Promotes Hemopoietic-Derived Angiogenic Cell Expansion and Arterial Specification}

\author{
The Scientific World Journal \\ Received 25 July 2018; Accepted 25 July 2018; Published 9 August 2018 \\ Copyright (c) 2018 The Scientific World Journal. This is an open access article distributed under the Creative Commons Attribution \\ License, which permits unrestricted use, distribution, and reproduction in any medium, provided the original work is properly \\ cited.
}

The Scientific World Journal and the authors have retracted the article titled "Inflammatory Microenvironment Promotes Hemopoietic-Derived Angiogenic Cell Expansion and Arterial Specification" [1]. The article was found to contain duplicated images from the authors' previous article in Blood, Zeoli et al. [2], after concerns were raised on PubPeer. Details of the duplication are as follows:

(1) pSTAT in Figure 7(d) in Blood and STAT in Figure 1 in The Scientific World Journal are the same, representing phosphorylated and unphosphorylated protein, respectively, and the $\beta$-actin panels are the same.

(2) Figure $1(\mathrm{Aa})$ in The Scientific World Journal is modified from Figure $4(\mathrm{Ci})$ in Blood, where the top of each image is the same, but parts of the top right of Figure $4(\mathrm{Ci})$ are duplicated in the bottom left of Figure 1(Aa).

(3) The lower panels of Figure 4(b) in Blood, when rotated left, are the same as the panels in Figure 1(c) in The Scientific World Journal. The middle panel in Blood is the same as that in The Scientific World Journal when the contrast and exposure are adjusted, representing Eph4 and EphrinB2, respectively.

The corresponding author Dr. Brizzi apologizes for this.

\section{References}

[1] P. Dentelli and M. F. Brizzi, "Inflammatory microenvironment promotes hemopoietic-derived angiogenic cell expansion and arterial specification," The Scientific World Journal, vol. 8, pp. 1111-1115, 2008.

[2] A. Zeoli, P. Dentelli, A. Rosso et al., "Interleukin-3 promotes expansion of hemopoietic-derived CD45+ angiogenic cells and their arterial commitment via STAT5 activation," Blood, vol. 112, no. 2, pp. 350-361, 2008. 


\title{
Inflammatory Microenvironment Promotes Hemopoietic-Derived Angiogenic Cell Expansion and Arterial Specification
}

\author{
Patrizia Dentelli and Maria Felice Brizzi* \\ Department of Internal Medicine, University of Torino, Corso Dogliotti 14, \\ 10126 Torino, Italy
}

E-mail: mariafelice.brizzi@unito.it

Received July 23, 2008; Revised September 30, 2008; Accepted October 13, 2008; Published November 2, 2008

KEYWORDS: endothelial progenitor cells (EPC), STAT5, IL-3, circulating angiogenic cells, inflammation, angiogenesis, arterial morphogenesis

Physiological and pathological angiogenesis, which occurs throughout adult life (adult angiogenesis), is mainly supported by sprouting cells and co-option of neighboring pre-existing vessels[1]. However, mature endothelial cells (EC) possess limited regenerative capacity[2]. The recent identification of bone marrow (BM)-derived circulating endothelial progenitor cells (EPC) has attracted interest into their role in maintaining vascular homeostasis[3]. The first description of putative EPC came from Asahara et al. in 1997[3]. From this initial report, intense efforts have focused on defining the role of circulating BMderived EPC in the repair of damaged vascular endothelium and on translating this information into human clinical trials. However, the limited efficacy uncovered by clinical studies led to the conclusion that a plurality of cell types, improperly defined as EPC, contribute to vascular remodeling in adult life. Recently, Hur et al.[4] described two different EPC subpopulations, denoted as early and late EPC. Early EPC, also denoted as circulating angiogenic cells (CAC), derive from peripheral blood mononuclear cells (PB-MNC) and express CD14 and CD45 surface markers. Because of their low proliferative rate and low differentiation capability[4,5], the therapeutic effect exerted by these cells seems to be most likely mediated by their secretory phenotype, rather than by their direct contribution to vessel growth[5]. Late EPC, on the other hand, arise exclusively from a CD14-negative subpopulation of PB-MNC, showing a high proliferative rate and the ability to form new vascular networks directly[4,6,7]. Accordingly, several studies suggested their origin from circulating angioblasts[8]. Thus, EPC appear as a heterogeneous group of cells, which originate from multiple precursors within the BM, which can be present in $\mathrm{PB}$ at different stages of endothelial differentiation.

To contribute to vessel formation, EPC need to be mobilized from the BM in order to home to the sites of ongoing vascular damage or tumor neovascularization, and to differentiate into mature EC[9]. Vascular endothelial growth factor (VEGF) and stromal cell-derived factor-1 (SDF-1), produced by hemopoietic-derived cells and infiltrating area of neovascularization, appear to be important mediators of EPC mobilization[10]. Despite several efforts, the mechanisms regulating EPC homing to sites of injury or active neoangiogenesis are poorly understood. Several lines of evidence suggest that the mechanisms involved in the recruitment of inflammatory cells within sites of inflammation might also regulate EPC homing. In particular, the inflammatory microenvironment seems to control the adhesive and signaling 
events[11]. In line with the role of inflammation in mediating EPC recruitment, we have recently characterized a novel adhesion/survival-promoting function of the membrane-bound Kit ligand into sites of active neoangiogenesis[12].

The positive contribution of hemopoietic-derived cells in inflammatory and tumor neoangiogenesis has been extensively documented[13]; however, their functions are still controversial. It seems that hemopoietic-derived cells, rather than directly contribute to neovessel formation, have a broader function in promoting angiogenesis[5,13]. We have previously shown that the hemopoietic growth factor IL-3, originally implicated in the survival, proliferation, and differentiation of hemopoietic progenitor/stem cells (HSC) and mature cells[14,15], also promotes in vivo angiogenesis[16]. Our finding is consistent with the observation that IL-3 is an activated T lymphocyte-derived cytokine[17], produced in such pathological conditions as inflammation and cancer, in which angiogenetic developmental processes are recapitulated. To shed light on the potential molecular events determinant for IL-3-mediated physiological and/or pathological regulation of vessel growth, our group has recently investigated its effect in controlling angiogenic cell proliferation, differentiation, and vascular specification. In particular, we have demonstrated that IL-3 promotes a rapid expansion of a subset of CAC, expressing the CD45 surface marker and a low frequency of CD14 (see Table 1)[18]. The observation that the genotyped CD45+ cells, isolated from Polycythemia Vera patients, bared the same JAK2 mutation as the pathological BM-derived cells confirmed their origin from HSC[19]. Moreover, when exposed to IL-3, these cells acquired endothelial commitment, denoted by the expression of endothelial cell markers, such as VEGF receptor-2 (KDR), CD31, and Tie-2 (Table 1), and by the ability to contribute to neoformed vessels directly, when added to cancer-derived EC injected into SCID mice (Fig. 1A and B).

\section{TABLE 1}

Characterization of Circulating and BM-Derived Angiogenic Cells Expanded in Response to IL-3

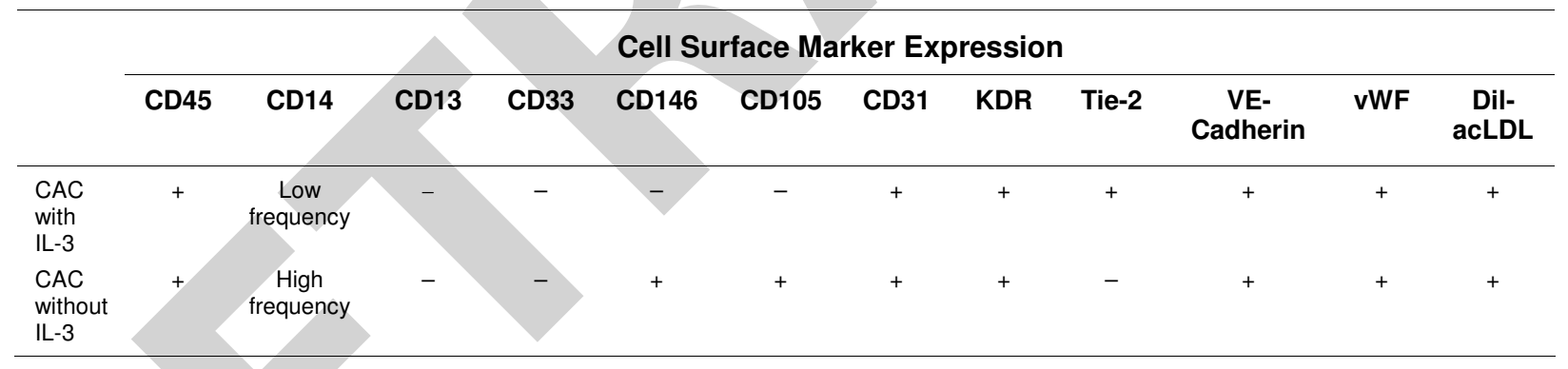

Cell surface markers expressed by circulating and BM-derived angiogenic cells cultured in the presence of IL-3 and compared to cell surface markers expressed by canonical CAC[6,7]. Abbreviation: vWF = von Willebrand factor.

EC diversity is the main determinant of vascular bed diversification during the developmental processes and the characterization of the pathways regulating these events has rapidly increased[20]. The genetic approach has revealed that Notch/VEGF signaling pathways have a central role in arterial/venous differentiation[21,22]. However, the evidence that EC arterial specification is maintained in the absence of Notch signal[23] suggests that signals outside the vasculature could also contribute to this process. In this regard, our study has shown that in the presence of an inflammatory stimulus, circulating and BMderived CD45+ angiogenic cells recapitulate, both in vitro and in vivo, arterial specification, as demonstrated by the specific expression of arterial markers (membrane ligand Ephrin B2, Notch1, and Notch4 receptors) (Fig. 1C). 

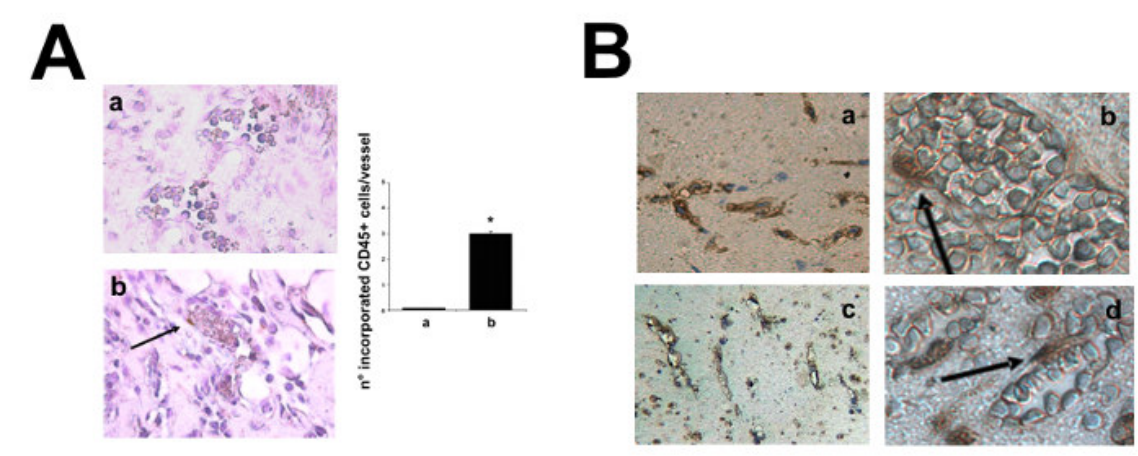

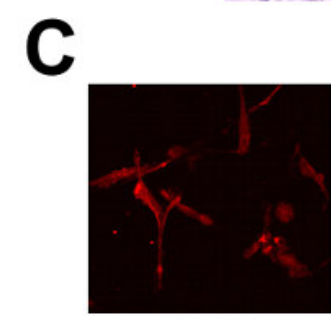

CD31

E
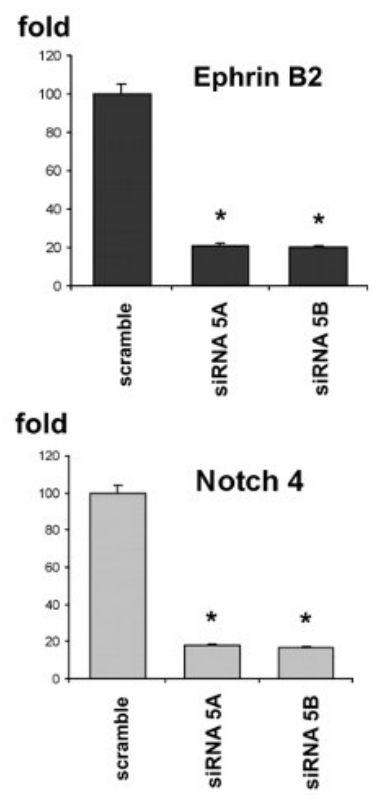

D

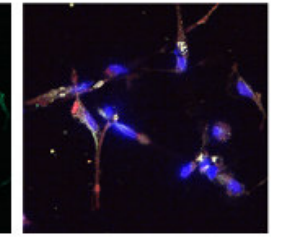

merge
EphrinB 2

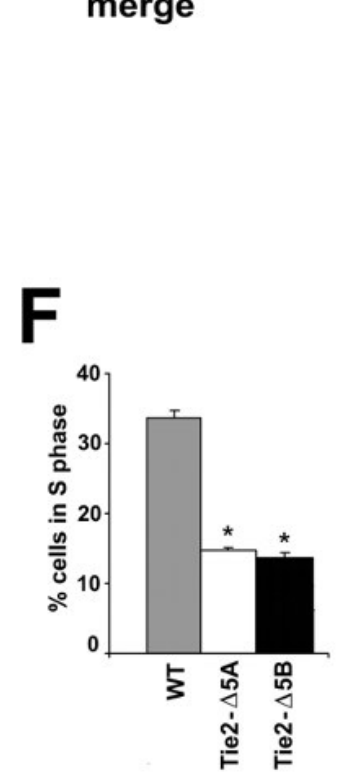

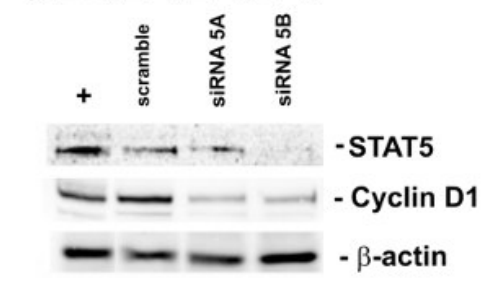

G

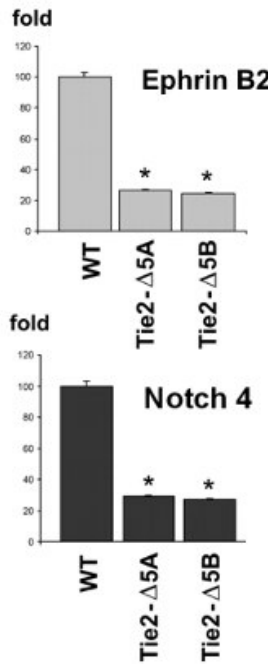

FIGURE 1. IL-3 promotes expansion of hemopoietic-derived CD45+ angiogenic cells and their arterial commitment via STAT5. (A) Tumor EC, added to Matrigel and injected into SCID mice, form functional neovessels (panel a). Prelabeled IL3-expanded cells, injected in the tail vein, are incorporated in tumor neovessels (panel b). Black arrows indicate the incorporated CD45+ cells. The number of incorporated cells per vessel is reported in the histogram. (B) IL-3-cultured CD45+ cells, added to Matrigel and injected into SCID mice, are able to form functional vessels, after 15 days (panels b,d). No functional vessels could be detected early after injection (5 days) (panel a,c). Black arrows indicate positive CD45+ cells. (C) Arterial specification is documented by the positive double staining (CD31, red; EphrinB2, green) of IL-3expanded CD45+ cells. DAPI staining is used as nuclear marker. (D) Depletion of STAT5 in IL-3-expanded CD45+ cells abrogates cyclin D1 expression. (E) STAT5 silencing also prevents arterial specification. (F,G) IL-3-mediated cell-cycle progression and arterial specification of BM-derived CD45+ cells is prevented in Tie-2-transgenic mice expressing dominant-negative STAT5 constructs. 
The expansion and differentiation of stem cells and progenitors are dynamic multistage processes, requiring sequential activation of particular signaling molecules[10]; however, the molecular determinants of these events are still poorly defined. IL-3 exerts its biological effects by activating the STAT5 signaling pathway, both in hemopoietic[24] and vascular tissues[16], as revealed by the intrinsic defective IL-3 responsiveness of STAT5-deficient HSC[25] and EC[26]. Similarly, in our model, STAT5, by acting as a signaling component and as a transcriptional factor controlling cyclin D1 expression, regulates cell-cycle progression and expansion of CD45+ angiogenic cells (Fig. 1D and E). In addition, by generating Tie2-transgenic mice[27], we demonstrated that STAT5 inactivation prevented IL-3sustained neovessel formation, IL-3-mediated cyclin D1 expression, and progression into the cell-cycle of BM-derived CD45+ cells, as well as their arterial specification (Fig. 1F and G).

Stem and progenitor cells that reside in an adaptive BM microenvironment readily respond to demand for revascularization by undergoing differentiation. However, whether this process initiates in BM or at the neoangiogenic sites is still controversial. In our model, where angiogenetic developmental processes are recapitulated, IL-3 provides a permissive environment for expansion and endothelial commitment of a subset of hemopoietic-derived progenitor cells.

Cell therapy efficacy is certainly dependent on the number, functional capability, and successful retention of cells in the site of action. It is also anticipated that greater numbers of surviving cells would translate into more paracrine stimuli, which result in greater biological responses. Several factors play a role in reducing cell bioavailability after cell-base therapy, including the absence of survival factors. We now have reason to believe that IL-3, released into the inflammatory microenvironment, can induce expansion and arterial specification of angiogenic cells, which may translate into a more efficient response to vascular dysfunction. In addition, our data enable the possibility to develop strategies for their ex vivo expansion and genetic modification, and to obtain tissue vascularization in clinical settings where angiogenesis might be required. Finally, the identification of the STAT5 signaling pathway, as the molecular signature of IL-3-mediated vasculogenic process, provides the rationale for therapeutically targeting STAT5 to hamper pathological angiogenesis.

\section{REFERENCES}

1. Carmeliet, P. (2005) Angiogenesis in life, disease and medicine. Nature 438, 932-936.

2. Caplan, B.A. and Schwartz, C.J. (1973) Increased endothelial cell turnover in areas of in vivo Evans blue uptake in the pig aorta. Atherosclerosis 17, 401-417.

3. Asahara, T., Murohara, T., Sullivan, A., Silver, M., van der Zee, R., Li, T., Witzenbichler, B., Schatteman, G., and Isner, J.M. (1997) Isolation of putative progenitor endothelial cells for angiogenesis. Science 275, 964-967.

4. Hur, J., Yoon, C., Kim, H.S., Choi, J.H., Kang, H.J., Hwang, K.K., Oh, B.H., Lee, M.M., and Park, Y.B. (2004) Characterization of two types of endothelial progenitor cells and their different contributions to neovasculogenesis. Arterioscler. Thromb. Vasc. Biol. 24, 288-293.

5. Rehman, J., Li, J., Orschell, C.M., and March, K.L. (2003) Peripheral blood "endothelial progenitor cells" are derived from monocyte/macrophages and secrete angiogenic factors. Circulation 107, 1164-1169.

6. Gulati, R., Jevremovic, D., Peterson, T.E., Chatterjee, S., Shah, V., Vile, R.G., and Simari, R.D. (2003) Diverse origin and function of cells with endothelial phenotype obtained from adult human blood. Circ. Res. 93, 1023-1025.

7. Ingram, D.A., Mead, L.E., Tanaka, H., Meade, V., Fenoglio, A., and Mortell, K. (2004) Identification of a novel hierarchy of endothelial progenitor cells using human peripherial and umbilical cord blood. Blood 104, 2752-2760.

8. Reyes, M., Dudek, A., Jahagirdar, B., Koodie, L., Marker, P.H., and Verfaillie, C.M. (2002) Origin of endothelial progenitors in human postnatal bone marrow. J. Clin. Invest. 109, 337-346.

9. Papayannopoulou, T. (2004) Current mechanistic scenarios in hematopoietic stem/progenitor cell mobilization. Blood 103, 1580-1585.

10. Rabbany, S.Y., Heissig, B., Hattori, K., and Rafii, S. (2003) Molecular pathways regulating mobilization of marrowderived stem cells for tissue revascularization. Trends Mol. Med. 9, 109-117.

11. Schenkel, A.R., Mamdouh, Z., and Muller, W.A. (2004) Locomotion of monocytes on endothelium is a critical step during extravasation. Nat. Immunol. 5, 393-400.

12. Dentelli, P., Rosso, A., Balsamo, A., Colmenares Benedetto, S., Zeoli, A., Pegoraro, M., Camussi, G., Pegoraro, L., and Brizzi, M.F. (2007) C-KIT, by interacting with the membrane-bound ligand, recruits endothelial progenitor cells to inflamed endothelium. Blood 109, 4264-4271.

13. De Palma, M. and Naldini, L. (2006) Role of haematopoietic cells and endothelial progenitors in tumour 
angiogenesis. Biochim. Biophys. Acta 1766, 159-166.

14. Greenberger, J.S., Eckner, R.J., Sakakeeny, M., Marks, P., Reid, D., Nabel, G., Hapel, A., Ihle, J.N., and Humphries, K.C. (1983) Interleukin 3-dependent hematopoietic progenitor cell lines. Fed. Proc. 42, 2762-2771.

15. Metcalf, D., Begley, C.G., Johnson, G.R., Nicola, N.A., Lopez, A.F., and Williamson, D.J. (1986) Effects of purified bacterially synthesized murine multi-CSF (IL-3) on hematopoiesis in normal adult mice. Blood 68, 46-57.

16. Dentelli, P., DelSorbo, L., Rosso, A., Molinar, A., Garbarino, G., Camussi, G., Pegoraro, L., and Brizzi, M.F. (1999) Human IL-3 stimulates endothelial cell motility and promotes in vivo new vessel formation. J. Immunol. 163, 21512159.

17. Dentelli, P., Rosso, A., Calvi, C., Ghiringhello, B., Garbarino, G., Camussi, G, Pegoraro, L., and Brizzi, M.F. (2004) IL-3 affects endothelial cell-mediated smooth muscle cell recruitment by increasing TGF beta activity: potential role in tumor vessel stabilization. Oncogene 23, 1681-1692.

18. Zeoli, A., Dentelli, P., Rosso, A., Togliatto, G., Trombetta, A., Damiano, L., di Celle, P.F., Pegoraro, L., Altruda, F., and Brizzi, M.F. (2008) Interleukin-3 promotes expansion of hemopoietic-derived CD45+ angiogenic cells and their arterial commitment via STAT5 activation. Blood 112, 350-361.

19. Yoder, M.C., Mead, L.E., Prater, D., Krier, TR, Mroueh, K.N., Li, F., Krasich, R., Temm, C.J., Prchal, J.T., and Ingram, D.A. (2007) Redefining endothelial progenitor cells via clonal analysis and hematopoietic stem/progenitor cell principals. Blood 109, 1801-1809.

20. Harvey, N.L. and Oliver, G. (2004) Choose your fate: artery, vein or lymphatic vessel? Curr. Opin. Genet. Dev. 14, 499-505.

21. Iso, T., Hamamori, Y., and Kedes, L. (2003) Notch signaling in vascular development. Arterioscler. Thromb. Vasc. Biol. 23, 543-553.

22. Visconti, R.P., Richardson, C.D., and Sato, T.N. (2002) Orchestration of angiogenesis and arteriovenous contribution by angiopoietins and vascular endothelial growth factor (VEGF). Proc. Natl. Acad. Sci. U. S. A. 99, 8219-8224.

23. Aranguren, X.L., Luttun, A., Clavel, C., Moreno, C., Abizanda, G., Barajas, M.A., Pelacho, B., Uriz, M., Araña, M., Echavarri, A., Soriano, M., Andreu, E.J., Merino, J., Garcia-Verdugo, J.M., Verfaillie, C.M., and Prósper, F. (2007) In vitro and in vivo arterial differentiation of human multipotent adult progenitor cells. Blood 109, 2634-2642.

24. Mui, A.L., Wakao, H., Harada, N., O'Farrell, A.M., and Miyajima, A. (1995) Interleukin-3, granulocyte-macrophage colony-stimulating factor, and interleukin-5 transduce signals through two forms of STAT5. J. Leukoc. Biol. 57, 799803.

25. Bradley, H.L., Hawley, T.S., and Bunting, K.D. (2002) Cell intrinsic defects in cytokine responsiveness of STAT5deficient hematopoietic stem cells. Blood 100, 3983-3989.

26. Defilippi, P., Rosso, A., Dentelli, P., Calvi, C., Garbarino, G., Tarone, G., Pegoraro, L., and Brizzi, M.F. (2005) \{beta\}1 Integrin and IL-3R coordinately regulate STAT5 activation and anchorage-dependent proliferation. J. Cell Biol. 168, 1099-1108.

27. Takakura, N., Huang, X.L., Naruse, T., Hamaguchi, I., Dumont, D.J., Yancopoulos, G.D., and Suda, T. (1998) Critical role of the TIE2 endothelial cell receptor in the development of definitive hematopoiesis. Immunity 9, 677686.

\section{This article should be cited as follows:}

Dentelli, P. and Brizzi, M.F. (2008) Inflammatory microenvironment promotes hemopoietic-derived angiogenic cell expansion and arterial specification. TheScientificWorldJOURNAL 8, 1111-1115. DOI 10.1100/tsw.2008.142. 